\title{
Acta Neurologica Belgica
}

\author{
Patrick Cras $^{1}$
}

Received: 15 February 2018 / Accepted: 16 February 2018 / Published online: 5 March 2018

(c) Belgian Neurological Society 2018

After joint and thorough neurological examination, both the former editor-in-chief Michel Van Zandijcke and myself declare Acta Neurologica Belgica to be in good health! The journal's scope is mostly clinical with some more basic neurological science papers that are likely to carry a broad interest. Yearly, more than 500 articles are submitted and after review and improvement about one-third are finally accepted for publication. Short contributions predominate in the form of 'letters to the editor' and Neuro-Images. Full length papers are less frequently submitted and fewer attain the level of novelty and quality that would lead to acceptance. Therefore, in the future, the editors will encourage the publication of original research results. We will have to be more selective in the acceptance of short communications and images. More and more contributions come from Asia and the Middle East. Especially Turkey and Iran have been major contributors from outside Europe. In the future, we will continue to encourage submissions from emerging countries. The editors are well supported by the publisher with a state-of-the-art editorial website aiding in the selection of reviewers and decisional system. This has allowed us to shorten the time from submission to the first decision to 36 days. Judging from the ever-increasing download of fulltext articles, the journal is well read. The shareable link is a powerful instrument to enhance readership of an interesting article the reader may encounter. As a result, the impact factor, Cite Score and h5 index (the number of papers that have been cited at least five times) have all increased in the past few years. As a measure of excellence, we will consider some form of promotion of authors of influential and highly downloaded papers. Provided there is support of involved scientific societies, we should also consider involving influential authors from emerging countries as associate editors. Lastly, the Journal's Board Members are selected on behalf

Patrick Cras

patrick.cras@uantwerpen.be

1 Born-Bunge Institute, University of Antwerp, Antwerp University Hospital, Wilrijkstraat 10, B-2650 Edegem, Belgium of their expertise and activity as researchers and teachers. Therefore, the editors would like to encourage board members to submit articles that could be of interest to a general audience. We would also like to invite Belgian neurologists to submit consensus statements and guidelines.

Finally, the editors would like to express their sincere gratitude to the reviewers who have, despite their busy schedules, found time to provide us with comments to improve the quality of the papers.

Patrick Cras, MD, PhD

Editor-in-chief

\section{Compliance with ethical standards}

Conflict of interest The author declare that they have no conflict of interest.

Ethical approval This article do not contain studies with human participants or animals by author.

Informed consent For this type of study formal consent is not required. 